\title{
Molecular Reactivity and Absorption Properties of Melanoidin Blue-G1 through Conceptual DFT
}

\author{
Juan Frau ${ }^{1,+}$ and Daniel Glossman-Mitnik $1,2, *,+$ \\ 1 Departament de Química, Universitat de les Illes Balears, 07122 Palma de Mallorca, Spain; juan.frau@uib.es \\ 2 Laboratorio Virtual NANOCOSMOS, Departamento de Medio Ambiente y Energía, \\ Centro de Investigación en Materiales Avanzados, Miguel de Cervantes 120, \\ Complejo Industrial Chihuahua, Chihuahua, 31136 Chih, Mexico \\ * Correspondence: daniel.glossman@cimav.edu.mx; Tel.: +52-614-439-1151 \\ + These authors contributed equally to this work.
}

Received: 18 February 2018; Accepted: 28 February 2018; Published: 2 March 2018

\begin{abstract}
This computational study presents the assessment of eleven density functionals that include CAM-B3LYP, LC-wPBE, M11, M11L, MN12L, MN12SX, N12, N12SX, wB97, wB97X and wB97XD related to the Def2TZVP basis sets together with the Solvation Model Density (SMD) solvation model in calculating the molecular properties and structure of the Blue-G1 intermediate melanoidin pigment. The chemical reactivity descriptors for the system are calculated via the conceptual Density Functional Theory (DFT). The choice of the active sites related to the nucleophilic, electrophilic, as well as radical attacks is made by linking them with the Fukui function indices, the electrophilic Parr functions and the condensed dual descriptor $\Delta f(\mathbf{r})$. The prediction of the maximum absorption wavelength tends to be considerably accurate relative to its experimental value. The study found the MN12SX and N12SX density functionals to be the most appropriate density functionals in predicting the chemical reactivity of the studied molecule.
\end{abstract}

Keywords: melanoidins; Blue-G1; conceptual DFT; chemical reactivity; dual descriptor; Parr function; maximum absorption wavelength

\section{Introduction}

In food science, the Maillard reaction is well known (named after the French Chemist who first described it): it consists of the reaction produced between a reducing sugar and an amino acid (the link of a small number of amino acids forms peptides, and at a higher number, they form proteins). When a reducing sugar reacts with a protein, a compound called a Schiff base is formed, and the accumulation of several of these compounds degrades proteins until they create Advanced Glycation End-products (AGEs) that originate fibrils that accumulate in the brain. It is hypothesized that when brain proteins degrade, they may cause degenerative diseases such as Alzheimer's, Parkinson's or diabetes.

The completion of the Maillard or nonenzymatic browning reaction leads to melanoidin formation. If reducing sugars are left to react with biological molecules under physiological conditions, a similar reaction occurs in a process referred to as glycation. Notably, glycation has a considerable influence on the aging process among living organisms. It also has an impact on the pathology of several diseases [1]. In recent times, our emphasis has been on understanding how glycation takes place and the chemical reactivity that reducing carbohydrates have with amino acids, as well as the peptides participating in the process, which are often linked to some diseases, such as Parkinson's, diabetes and Alzheimer's [2-32].

The main feature notable in the Maillard reaction includes color formation. However, little is known about the colored moieties that cause the coloration. The light-weight intermediate colored products are referred to as Colored Maillard Reaction Products (CMRP), and it is possible to measure 
color production based on absorbance readings that the visible region of the spectrum indicates. The typical wavelengths that apply in measurements include 360 and $420 \mathrm{~nm}$ [33]. The CMRPs attract interest from the food industry, as well as other industries because they are hypothesized as having the potential of being photosensitizers, in which the can be employed as antioxidants associated with health, as well as colorants that dye-sensitized solar cells can use to produce alternative energy. These potential applications explain the focus on their chemical properties, with their molecular reactivity experiencing particular interest. Blue-G1 is among the interesting CMRPs that have been studied using conceptual DFT [34]. As a blue pigment, it is isolated from the reaction system involving D-glucose and glycine. This prompts the assumption that Blue-G1 may be of interest in applying the concepts of density functional theory in studying the chemical reactivity that the blue pigment exhibits.

Conceptual Density Functional Theory (DFT), which is also called chemical reactivity theory [2-32], provides a powerful tool that can be used in predicting, analyzing and interpreting the outcomes that chemical reactions generate [35-38]. Parr et al. [35] pioneered the coverage of the topic. However, several useful concepts that followed their work result from the analysis done on the density of molecular systems using the DFT. The concepts enable researchers to qualitatively predict how the chemical reactivity will take place in a given system. It is also possible to quantify the concepts. Collectively, the concepts would be referred to as conceptual DFT descriptors.

A new theory applicable in studying reactivity in organic chemistry was presented by Luis $R$. Domingo [39]. The theory is known as Molecular Electron Density Theory (MEDT) and proposes that the capability of the changes in electron density affects the molecular reactivity [39], and the way electron density is distributed at the ground determines the physical, as well as the chemical molecular attributes. MEDT encompasses DFT and also defines a number of new concepts and reactivity descriptors including the nucleophilicity N index, the Global Electron Density Transfer (GEDT), as well as the local condensed descriptors like the electrophilic $\mathrm{P}_{k}^{+}$and nucleophilic $\mathrm{P}_{k}^{-}$Parr functions [40]. Gradually, Parr functions are growing in popularity in efforts to understand organic chemistry processes. Several recent publications on Diels-Alder, as well as cycloaddition reactions evidence the rising popularity [41-43].

The Kohn-Sham theory involves calculating the molecular density, the energy that the system has and the orbital energies, particularly those associated with frontier orbitals including HOMO and LUMO [44-49]. The theory would be necessary in establishing the quantitative values of the various conceptual DFT descriptors. Interest in using Range-Separated (RS) exchange correlation functionals in Kohn-Sham DFT has been on the rise recently [50]. The functionals tend to partition the $\mathrm{r}_{12}^{-1}$ operator and exchange them into long- and short-range parts, whose range separation parameter, $\omega$, controls the rate of attaining the long-range behavior. It is possible to fix the value of $\omega$. The value can also be "tuned" through a system-by-system mechanism that minimizes some tuning norm. The basis of the optimal tuning approach is the knowledge that the energy that the Highest Occupied Molecular Orbital (HOMO) should have, $\epsilon_{H}(\mathrm{~N})$, in exact Kohn-Sham (KS), as well as Generalized $\mathrm{KS}$ theory for an $\mathrm{N}$ electron system ought to be exactly $-\mathrm{IP}(\mathrm{N})$. Hereby, IP represents the vertical ionization potential that is calculated considering a particular functional, the energy difference $\mathrm{E}(\mathrm{N}-1)$ $-\mathrm{E}(\mathrm{N})$. If approximate functionals are used, it would be possible to have considerable differences between $\epsilon_{H}(\mathrm{~N})$ and $-\mathrm{IP}(\mathrm{N})$. Optimal tuning constitutes determining a system-specific range-separation parameter $\omega$ non-empirically in an RSEfunctional. Optionally, it also implied that several other parameters including $\epsilon_{H}(\mathrm{~N})=-\mathrm{IP}(\mathrm{N})$ are satisfied optimally [51]. Even though no equivalency exists to match this prescription of the Electron Affinity (EA) coupled with LUMO in the case of neutral species, it is possible that $\epsilon_{H}(\mathrm{~N}+1)=-\mathrm{EA}(\mathrm{N})$, which facilitates the finding of an optimized value of $\omega$ that is then optimized to establish both properties. Such would make it easy to predict the conceptual DFT descriptors. The simultaneous prescription has in the past been referred to as the "KIDprocedure", courtesy of the analogy it shares with Koopmans' theorem [2-32].

This implies that the appropriateness that a particular density functional has in making a prediction on the conceptual DFT descriptors directly by relying on the property that the neutral 
molecule can be easily estimated. It only requires one to check the way it has followed the KID procedure. Nevertheless, tune optimization depends on the system and had to be performed for each molecule one at a time. Therefore, examining the various density functionals exhibiting significant accuracy across various types of databases in physics, chemistry, as well as where the $\omega$ value is fixed is done to determine how to perform the practical technique.

This study seeks to undertake a comparative study of the way a number of recent density functionals perform in reproducing the chemical reactivity descriptors that the Blue-G1 pigment has in the KID formalism to have sufficient insights about their molecular attributes that future studies use on the chemical reactivity that colored melanoidins of larger molecular weights that form from the reaction that reducing sugars have with proteins and peptides.

\section{Theoretical Background}

The theoretical background of this study is similar to the previous conducted research presented [2-32] and will be shown here to be complete, because this research is a component of a major project that is in progress.

If we consider the KID procedure presented in our previous works [2-32] together with a finite difference approximation, then the global reactivity descriptors can be written as [36,52-55] :

$$
\begin{array}{cc}
\text { Electronegativity } & \chi=-\frac{1}{2}(I+A) \approx \frac{1}{2}\left(\epsilon_{L}+\epsilon_{H}\right) \\
\text { Global hardness } & \eta=(I-A) \approx\left(\epsilon_{L}-\epsilon_{H}\right) \\
\text { Electrophilicity } & \omega=\frac{\mu^{2}}{2 \eta}=\frac{(I+A)^{2}}{4(I-A)} \approx \frac{\left(\epsilon_{L}+\epsilon_{H}\right)^{2}}{4\left(\epsilon_{L}-\epsilon_{H}\right)} \\
\text { Electron-donating power } & \omega^{-}=\frac{(3 I+A)^{2}}{16(I-A)} \approx \frac{\left(3 \epsilon_{H}+\epsilon_{L}\right)^{2}}{16 \eta} \\
\text { Electron-accepting power } & \omega^{+}=\frac{(I+3 A)^{2}}{16(I-A)} \approx \frac{\left(\epsilon_{H}+3 \epsilon_{L}\right)^{2}}{16 \eta} \\
\text { Net electrophilicity } & \Delta \omega^{ \pm}=\omega^{+}-\left(-\omega^{-}\right)=\omega^{+}+\omega^{-}
\end{array}
$$

where $\epsilon_{H}$ and $\epsilon_{L}$ are the energies of the Highest Occupied and the Lowest Unoccupied Molecular Orbitals (HOMO and LUMO), respectively.

Applying the same ideas, the definitions for the local reactivity descriptors are [37,52,56-63]

$$
\begin{array}{cc}
\text { Nucleophilic Fukui function } & f^{+}(\mathbf{r})=\rho_{N+1}(\mathbf{r})-\rho_{N}(\mathbf{r}) \\
\text { Electrophilic Fukui function } & f^{-}(\mathbf{r})=\rho_{N}(\mathbf{r})-\rho_{N-1}(\mathbf{r}) \\
\text { Dual descriptor } & \Delta f(\mathbf{r})=\left(\frac{\partial f(\mathbf{r})}{\partial N}\right)_{v(\mathbf{r})} \\
\text { Nucleophilic Parr function } & P^{-}(\mathbf{r})=\rho_{s}^{r c}(\mathbf{r}) \\
\text { Electrophilic Parr function } & P^{+}(\mathbf{r})=\rho_{s}^{r a}(\mathbf{r})
\end{array}
$$

where $\rho_{N+1}(\mathbf{r}), \rho_{N}(\mathbf{r})$ and $\rho_{N-1}(\mathbf{r})$ are the electronic densities at point $\mathbf{r}$ for the system with $N+1$, $N$ and $N-1$ electrons, respectively, and $\rho_{s}^{r c}(\mathbf{r})$ and $\rho_{s}^{r a}(\mathbf{r})$ are related to the Atomic Spin Density (ASD) at the $\mathbf{r}$ atom of the radical cation or anion of a given molecule, respectively [40].

\section{Settings and Computational Methods}

Consistent with the work presented earlier [2-32], this study involved performing computational studies with the Gaussian 09 series of programs [64] whose density functional methods are implemented in the computational package. The gradient technique was relied upon to determine 
the equilibrium geometries of the molecules; whereas the determination of the force constants and vibrational frequencies involves computing analytical frequencies on various stationary points that are obtained as optimization is completed to check whether the minima were real. The basis set that this work used included Def2SVP for both geometry optimization, as well as frequencies; whereas Def2TZVP was involved in calculating the electronic characteristics $[65,66]$.

Eleven density functionals were selected to calculate the molecular structure and properties that the studied systems have. The selected functionals offer satisfactory results consistently in relation to a number of thermodynamic and structural attributes. Among the functionals are included CAM-B3LYP, which entails Handy and co-workers' long-range-corrected version of B3LYP done through the Coulomb-attenuating method [67]. Another functional relates to LC-wPBEconstituting the long-range-corrected wPBE density functional [68]. In addition, M11entails a range-separated hybrid meta-Generalized Gradient Approximation (GGA) [69]. M11L, on the other hand, is a dual-range local meta-GGA [70], while MN12L consists of a non-separable local meta-Non-separable Gradient Approximation (NGA) [71]. MN12SX entails a range-separated hybrid non-separable meta-NGA [72], while N12 is an NGA [73]. N12SX comprise of a range separated hybrid NGA [72] as the wB97 and wB97X long-range corrected density functionals [74] together with the wB97XD version include empirical dispersion [75]. GGA in these functionals represents the generalized gradient approximation whereby the density functional is dependent on the up and down spin densities together with their reduced gradient; whereas NGA denotes the non-separable gradient approximation in which the density functional tends to rely on the up and down spin densities, as well as their lowered gradient, while also assuming a non-separable form. The various calculations were done with water being the solvent. Integral Equation Formalism-Polarized Continuum Model (IEF-PCM) computations were involved as the Solvation Model Density (SMD) provided [76].

\section{Results and Discussion}

This study took the molecular structure of the Blue-G1 intermediate melanoidin pigment from PubChem (https: / / pubchem.ncbi.nlm.nih.gov), a website that acts as the public repository for information pertaining to chemical substances together with the biological activities with which they are associated. The molecular structure with the IUPAC (International Union of Pure and Applied Chemistry) name includes 5-[1,4-bis-carboxymethyl-5-(2,3,4-trihydroxybutyl)-1,4-dihydropyrrolo[3,2-b]pyrrol-2-ylmethyl-ene]1,4-bis-carboxymethyl-2-(2,3,4-trihydroxybutyl)-4,5-dihydropyrrolo[3,2-b]pyrrol-1-ium [34]. The preoptimization of the resultant system involved selecting the most stable conformers. The selection was done using random sampling, which involved molecular mechanics techniques and inclusion of the various torsional angles via the general MMFF94force field [77-81], which involves the Marvin View 17.15 program, which constitutes an advanced chemical viewer suited to multiple and single chemical queries, structures and reactions (https:/ / www.chemaxon.com). Afterwards, the structure that the resultant lower-energy conformer assumes was reoptimized using the eleven density functionals previously mentioned in the previous section together with the Def2SVP basis set, as well as the SMD solvation model in which the solvent was water.

The analysis of the results obtained in the study aimed at verifying that the KID procedure was fulfilled. Upon doing this previously, several descriptors associated with the results that HOMO and LUMO calculations obtained are related to the results obtained using the vertical I and $\mathrm{A}$ following the $\triangle S C F$ procedure. A link exists between the three main descriptors and the simplest conformity to Koopmans' theorem by linking $\epsilon_{H}$ with -I, $\epsilon_{L}$ with -A, and their behavior in describing the HOMO-LUMO gap as $J_{I}=\left|\epsilon_{H}+E_{g S}(N-1)-E_{g S}(N)\right|, J_{A}=\left|\epsilon_{L}+E_{g S}(N)-E_{g S}(N+1)\right|$ and $J_{H L}=\sqrt{J_{I}^{2}+J_{A}^{2}}$. Notably, the $J_{A}$ descriptor is an approximation that is only valid if the HOMO of the radical anion (the SOMO) shares similarity with the LUMO of the neutral system. Consequently, we decided to design another descriptor $\Delta \mathrm{SL}$, to guide in verifying how the approximation is accurate.

Table 1 illustrates the electronic energies of neutral, positive and negative molecular systems of Blue-G1, HOMO, LUMO and SOMO orbital energies (all in au), while the calculation of $J_{I}, J_{A}$, 
$J_{H L}$ and $\triangle S L$ descriptors involves using the eleven density functionals, as well as the Def2TZVP basis set that would use water as a solvent that is simulated through the SMD parametrization of the IEF-PCM model.

Table 1. Electronic energies of the neutral, positive and negative molecular systems (in au) of Blue-G1, the HOMO, LUMO and SOMO orbital energies (also in au), $J_{I}, J_{A}, J_{H L}$ and $\triangle$ SLdescriptors calculated with the eleven density functionals and the Def2TZVPbasis set using water as as solvent simulated with the Solvation Model Density (SMD) parametrization of the Integral Equation Formalism-Polarized Continuum Model (IEF-PCM) model.

\begin{tabular}{ccccccccccc}
\hline & Eo & E+ & E- & HOMO & LUMO & SOMO & J(I) & J(A) & J(HL) & $\Delta$ HL \\
\hline CAM-B3LYP & -2395.4460 & -2395.2561 & -2395.5734 & -0.2380 & -0.0783 & -0.1748 & 0.0480 & 0.0490 & 0.0686 & 0.0965 \\
LC-wBPE & -2395.0237 & -2394.8290 & -2395.1654 & -0.2812 & -0.0560 & -0.2234 & 0.0865 & 0.0857 & 0.1217 & 0.1674 \\
M11 & -2395.2791 & -2395.0804 & -2395.4158 & -0.2761 & -0.0603 & -0.2104 & 0.0774 & 0.0764 & 0.1087 & 0.1501 \\
M11L & -2395.2507 & -2395.0467 & -2395.3823 & -0.1974 & -0.1406 & -0.1235 & 0.0066 & 0.0090 & 0.0112 & 0.0171 \\
MN12L & -2394.3267 & -2394.1354 & -2394.4448 & -0.1850 & -0.1254 & -0.1122 & 0.0063 & 0.0073 & 0.0097 & 0.0132 \\
MN12SX & -2394.4578 & -2394.2578 & -2394.5853 & -0.1995 & -0.1271 & -0.1277 & 0.0004 & 0.0004 & 0.0006 & 0.0006 \\
N12 & -2396.1437 & -2395.9620 & -2396.2534 & -0.1732 & -0.1199 & -0.1013 & 0.0086 & 0.0102 & 0.0133 & 0.0186 \\
N12SX & -2395.4309 & -2395.2397 & -2395.5531 & -0.1926 & -0.1199 & -0.1239 & 0.0014 & 0.0023 & 0.0027 & 0.0040 \\
wB97 & -2396.1313 & -2395.9395 & -2396.2668 & -0.2774 & -0.0513 & -0.2165 & 0.0856 & 0.0842 & 0.1201 & 0.1652 \\
wB97X & -2395.9266 & -2395.7344 & -2396.0603 & -0.2715 & -0.0546 & -0.2102 & 0.0793 & 0.0791 & 0.1120 & 0.1556 \\
wB97XD & -2395.7877 & -2395.5925 & -2395.9205 & -0.2619 & -0.0643 & -0.1995 & 0.0668 & 0.0685 & 0.0957 & 0.1353 \\
\hline
\end{tabular}

Afterwards, the study focuses on the other four descriptors analyzing the effectiveness of the density functional in predicting the electronegativity $\chi$, the global electrophilicity $\omega$, the global hardness $\eta$ and for a combination of the conceptual DFT descriptors, taking into account the energies that the LUMO and HOMO or the vertical I and A are related; $J_{\chi}=\left|\chi-\chi_{K}\right|, J_{\eta}=\left|\eta-\eta_{K}\right|, J_{\omega}=$ $\left|\omega-\omega_{K}\right|$, and $J_{C D F T}=\sqrt{J_{\chi}^{2}+J_{\eta}^{2}+J_{\omega}^{2}}$, where CDFT stands for Conceptual DFT.

Next, we consider four other descriptors that analyze how useful the studied density functionals are for the prediction of the electronegativity $\chi$, the global hardness $\eta$, the global electrophilicity $\omega$ and for a combination of these conceptual DFT descriptors, considering only the energies of the HOMO and LUMO or the vertical I and A: $J_{\chi}=\left|\chi-\chi_{K}\right|, J_{\eta}=\left|\eta-\eta_{K}\right|, J_{\omega}=\left|\omega-\omega_{K}\right|$, and $J_{C D F T}=\sqrt{J_{\chi}^{2}+J_{\eta}^{2}+J_{\omega}^{2}}$, where CDFT stands for Conceptual DFT. The results of the calculations of $J_{\chi}, J_{\eta}, J_{\omega}$ and $J_{C D F T}$ for the Blue-G1 intermediate melanoidin pigment are displayed in Table 2.

Table 2. $J_{\chi}, J_{\eta}, J_{\omega}$ and $J_{C D F T}$ for the Blue-G1 intermediate melanoidin pigment.

\begin{tabular}{ccccc}
\hline & $\mathbf{J}_{\chi}$ & $\mathrm{J}_{\boldsymbol{\eta}}$ & $\mathrm{J}_{\boldsymbol{\omega}}$ & $\mathrm{J}_{\boldsymbol{C} \boldsymbol{D} \boldsymbol{F} \text { T }}$ \\
\hline CAM-B3LYP & 0.0005 & 0.0970 & 0.1226 & 0.1564 \\
LC-wBPE & 0.0004 & 0.1722 & 0.2035 & 0.2666 \\
M11 & 0.0005 & 0.1538 & 0.1610 & 0.2226 \\
M11L & 0.0012 & 0.0156 & 0.0568 & 0.0590 \\
MN12L & 0.0005 & 0.0136 & 0.0387 & 0.0410 \\
MN12SX & 0.0004 & 0.0000 & 0.0009 & 0.0010 \\
N12 & 0.0008 & 0.0188 & 0.0544 & 0.0575 \\
N12SX & 0.0004 & 0.0037 & 0.0101 & 0.0108 \\
wB97 & 0.0007 & 0.1698 & 0.1783 & 0.2462 \\
wB97X & 0.0001 & 0.1583 & 0.1654 & 0.2290 \\
wB97XD & 0.0009 & 0.1353 & 0.1483 & 0.2008 \\
\hline
\end{tabular}

As Table 1 provides, the KID procedure applies accurately for MN12SX and N12SX density functionals that are range-separated hybrid meta-NGA, as well as range-separated hybrid NGA density functionals, respectively. In fact, the values of $\mathrm{J}_{I}, \mathrm{~J}_{A}$ and $\mathrm{J}_{H L}$ are actually not zero. Nevertheless, the results tend to be impressive especially for the MN12SXdensity functional. In addition, the $\Delta$ SL descriptor reaches the minimum values when the MN12SX and N12SX density functionals are used in 
the calculations. This implies that there are sufficient justifications to assume that the LUMO of the neutral approximates the electron affinity.

It can be seen that the same density functional follows the KID procedure in the rest of the descriptors such as $J_{\chi}, J_{\eta}, J_{\omega}$ and $J_{D 1}$. The significance of these results is attributable to their illustration that reliance on $J_{I}, J_{A}$ and $J_{H L}$ would not be sufficient. For instance, if $J_{\chi}$ were considered on its own to apply to each density functional covered in this study, the values would considerably be near zero. In the case of the other descriptors, only the MN12SX and N12SX density functionals exhibit this behavior. This implies that the results that $\mathrm{J}_{\chi}$ record are likely to be because of the cancellation of errors by chance.

The Blue-G1 intermediate melanoidin pigment would be better studied using the Time-Dependent Density Functional Theory (TDDFT) because the pigment is a colored molecule. Various TDDFT studies of different sizes of molecules have used the optimally-tuned RSH density functionals. The studies report that absorption properties improve especially when applying the charge-transfer phenomenon [51,82-100].

The considerable success of the approach is however undermined by the issue of tuning being system dependent. Therefore, focus should be on establishing the effectiveness of the behaviors of the fixed RSH density functionals in describing the excitation characteristics. In his works, Becke has recently mentioned that the adiabatic connection and the ideas of Hohenberg, Kohn and Sham apply only to electronic ground states comprise a common misconception [101]. Furthermore, consistent with Baerends et al., the KS model is not appreciated for being superior because of its lowest excitation energy in molecules. Physically, it amounts to an excitation of the KS system rather than electron addition, as would be the case in Hartree-Fock. Thus, it can effectively be used as a measure of the optical gap and is an effective approximation to the gap (in molecules) [102]. In their conclusion, van Meer et al. advance that the HOMO-LUMO gap associated with the KS model tends to be an approximation of the lowest excitation energy, a desirable characteristic with no concerns regarding it [103].

Therefore, calculation of the maximum wavelength absorption of the Blue-G1 pigment involved conducting TDDFT calculations with the aforementioned eleven density functionals at the same level of model chemistry and theory as the calculations for establishing the ground state of the molecule. Table S1 of the Supplementary Information presents the results by comparing the values involved in the ground-state approximation derived from the HOMO-LUMO gap, as well as the TDDFT ones together with the experimental value of $629 \mathrm{~nm}$ [34]. Moreover, Figure 1 provides an illustration that compares the results graphically.

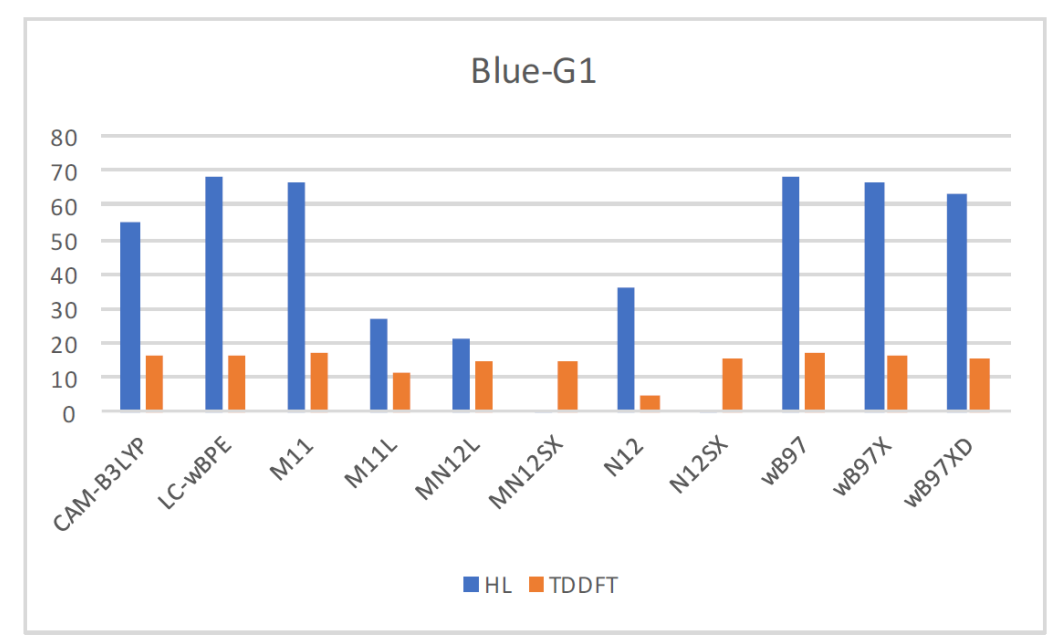

Figure 1. A graphical comparison of the results for the calculation of the $\lambda_{\max }$ of the Blue-G1 pigment between the HOMO-LUMO gap prediction, the TDDFT values and the experimental data. 
Notably, the presented results suggest that the differences with the experimental value for $\lambda_{\text {max }}$ tend to have the same order in the various functionals that the current study considers, apart from the N12 density functional. If the $\lambda_{\max }$ values that the HOMO-LUMO gap generates were the ones considered, MN12SX and N12SX would appear to be accurate especially in predicting this value. This does not apply in the rest of the density functionals that the study considers. In fact, the results that the TDDFT calculations give can be improved by expanding the basis set and integrating vibronic corrections; despite the level of accuracy in predicting the excited state property being remarkable if calculations are made at the ground state with the suitable choice of density functional.

Upon verifying that the MN12SX density functional is the best suited for the calculation of the global reactivity density descriptors and in predicting the $\lambda_{\max }$ in agreement with the experimental, Figure 2 presents the optimized structure o the Blue-G1 pigment graphically as calculated based on the theory; whereas Tables S2 and S3 of the Supplementary Materials illustrate the bond length and the bond angles.

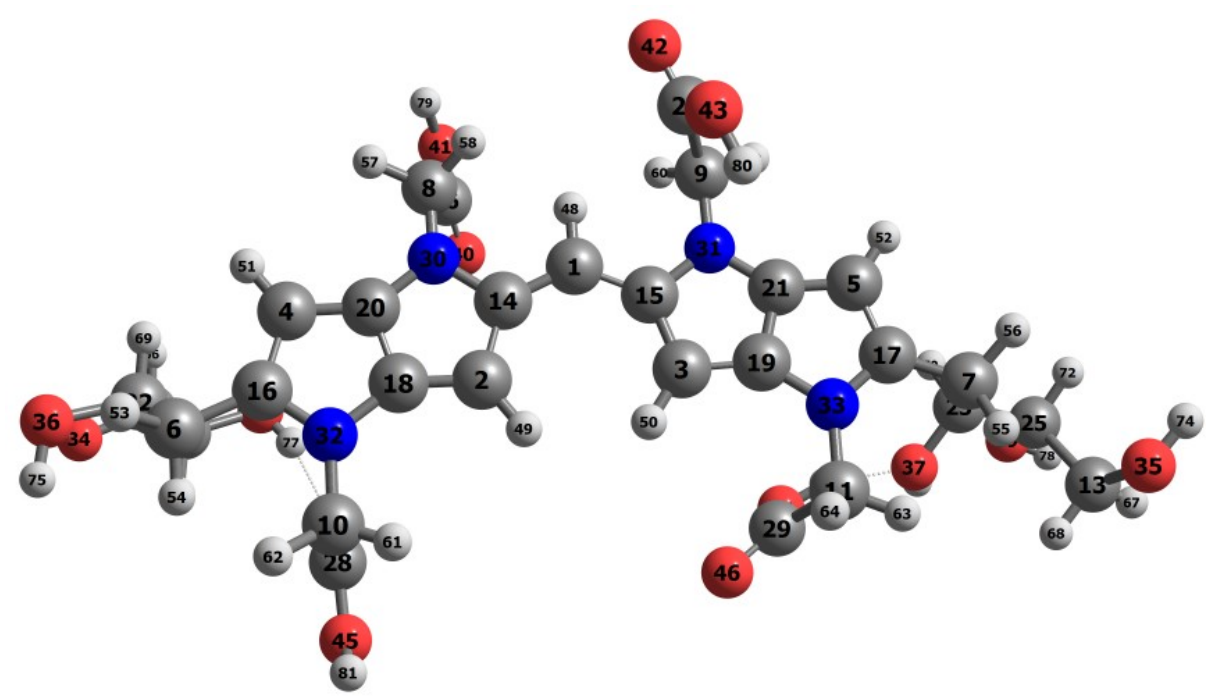

Figure 2. An schematic representation of the optimized structure of the Blue-G1 pigment calculated with the MN12SX density functional showing the numbering of the atoms.

Table 3 illustrates the results obtained after calculating the electronegativity $\chi$, chemical hardness $\eta$, global electrophilicity $\omega$, electron-accepting $\left(\omega^{+}\right)$and electron-donating $\left(\omega^{-}\right)$powers, as well as net electrophilicity powers with the MN12SX density. The Def2TZVP basis set is used with water acting as a solvent in line with the SMD solvation model.

Table 3. Global reactivity descriptors for the Blue-G1 intermediate melanoidin pigment calculated with the MN12SX density functional.

\begin{tabular}{ccc}
\hline Electronegativity $(\alpha)$ & Chemical Hardness $(\eta)$ & Electrophilicity $(\omega)$ \\
\hline 4.4428 & 1.9956 & 4.9457 \\
\hline Electron-Donating Power $\left(\omega^{-}\right)$ & Electron-Accepting Power $\left(\omega^{+}\right)$ & Net Electrophilicity $\left(\Delta \omega^{ \pm}\right)$ \\
\hline 7.1959 & 5.7429 & 12.9388 \\
\hline
\end{tabular}

The calculations of the condensed Fukui functions and dual descriptor are done by using the Chemcraft molecular analysis program to extract the Mulliken and NPAatomic charges [104] beginning with single-point energy calculations involving the MN12SX density functional that uses the Def2TZVP basis set. In line with the SMD solvation model, water is utilized as a solvent. 
Considering the potential application of the Blue-G1 molecule as an antioxidant, it is of interest to get insight into the active sites for radical attack. A graphical representation of the radical Fukui function $f^{0}$ is presented in Figure 3.

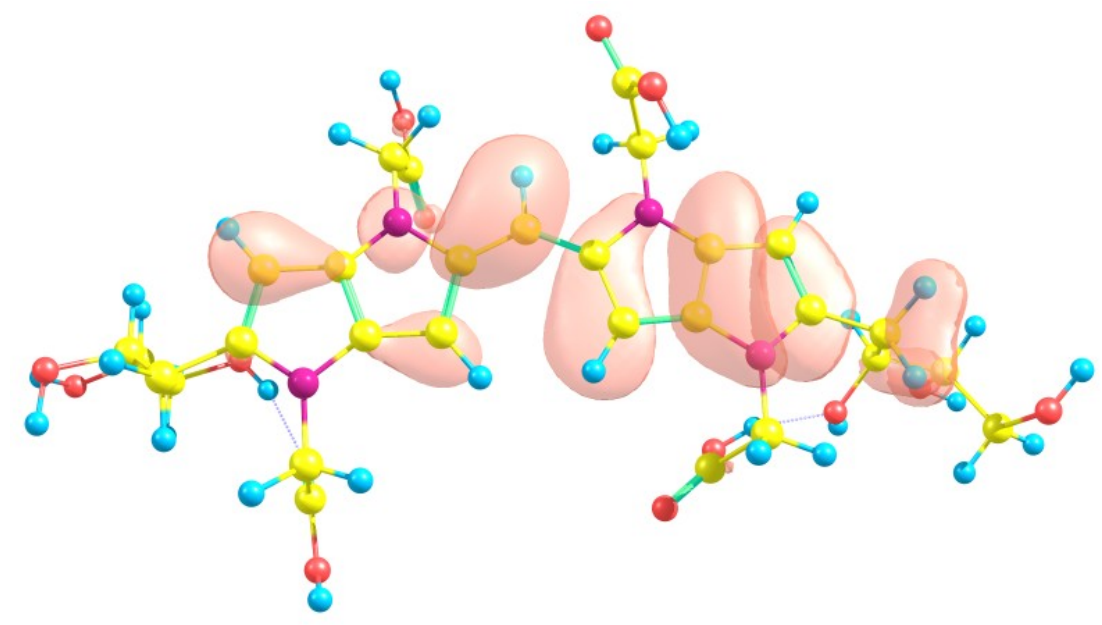

Figure 3. A graphical schematic representation of the radical Fukui function $f^{0}$ of the Blue-G1 intermediate melanoidin pigment.

The condensed electrophilic and nucleophilic Parr functions $P_{k}^{+}$and $P_{k}^{-}$over the atoms of the Blue-G1 pigment (excluding the $\mathrm{H}$ atoms) have been calculated by extracting the Mulliken and Hirshfeld (or CM5) atomic charges using the Chemcraft molecular analysis program [104] starting from single-point energy calculations of the ionic species with the MN12SX density functional using the Def2TZVP basis set in the presence of water as a solvent according to the (SMD) solvation model.

The results for the condensed dual descriptor calculated with Mulliken atomic charges $\Delta \mathrm{f}_{k}(\mathrm{M})$, with NPA atomic charges $\Delta \mathrm{f}_{k}(\mathrm{~N})$, the electrophilic and nucleophilic Parr functions with Mulliken atomic charges $P_{k}^{+}(\mathrm{M})$ and $P_{k}^{-}(\mathrm{M})$ and the electrophilic and nucleophilic Parr functions with Hirshfeld (or CM5) atomic charges $P_{k}^{+}(\mathrm{H})$ and $P_{k}^{-}(\mathrm{H})$ are displayed in Table 4.

From the results for the local descriptors in Table 4, it can be concluded that $\mathrm{C} 1$ will be the preferred site for a nucleophilic attack and that this atom will act as an electrophilic species in a chemical reaction. In turn, it can be appreciated that C14 and C15 will be prone to electrophilic attacks and that this atomic sites will act as nucleophilic species in chemical reactions where the Blue-G1 molecule is involved.

It has been already pointed out that although condensed Fukui functions give interesting results, they are not conclusive. In particular, it has been found that when studying metallic clusters, the condensed Fukui functions can predict the results of nucleophilic and electrophilic interactions with poor reliability [105]. However, from the results obtained in our work, we can present four important considerations: (i) in the first place, it is not the same to obtain conclusions about the reliability of the condensed Fukui functions when studying metallic clusters or even solid systems than when considering pure organic molecules, as happens in our case; (ii) the reliability of the results that we have obtained is impressive because the condensed Fukui functions (and thus the dual descriptor) have been calculated using two different schemes for the partition of the electronic density (i.e., the atomic charges), and the same has been done for the Parr functions: the conclusions about the reaction sites are exactly the same; (iii) in our work, we considered and presented the calculation of the dual descriptor rather than the condensed Fukui functions, and it has been shown by Martínez-Araya that the dual descriptor is more reliable for predicting the electrophilic and nucleophilic sites than the condensed Fukui functions [106]; (iv) as we have shown in several previous works [2-32], the reliability of the conceptual DFT descriptors for predicting the reactive sites of a given molecular system is heavily 
dependent on the goodness of the model chemistry employed for the calculations where we understand for goodness the ability to fulfill the KID procedure mentioned in the Introduction section.

Table 4. The condensed dual descriptor calculated with Mulliken atomic charges $\Delta \mathrm{f}_{k}(\mathrm{M})$ and with NPA atomic charges $\Delta \mathrm{f}_{k}(\mathrm{~N})$, the electrophilic and nucleophilic Parr functions with Mulliken atomic charges $P_{k}^{+}(\mathrm{M})$ and $P_{k}^{-}(\mathrm{M})$ and the electrophilic and nucleophilic Parr functions with Hirshfeld (or CM5) atomic charges $P_{k}^{+}(\mathrm{H})$ and $P_{k}^{-}(\mathrm{H})$ for the Blue-G1 molecule.

\begin{tabular}{|c|c|c|c|c|c|c|}
\hline Atom & $\Delta \mathbf{f}_{k}(\mathbf{M})$ & $\Delta \mathbf{f}_{k}(\mathbf{N})$ & $\mathbf{P}_{k}^{+}(\mathbf{M})$ & $\mathbf{P}_{k}^{-}(\mathbf{M})$ & $\mathbf{P}_{k}^{+}(\mathbf{H})$ & $\mathbf{P}_{k}^{-}(\mathbf{H})$ \\
\hline $1 C$ & 21.86 & 15.00 & 0.4112 & -0.1674 & 0.2228 & -0.0445 \\
\hline $2 \mathrm{C}$ & 9.23 & 8.64 & 0.1947 & -0.0404 & 0.1207 & 0.0106 \\
\hline $3 C$ & 8.05 & 7.72 & 0.1928 & -0.0218 & 0.1169 & 0.0226 \\
\hline $4 C$ & -6.14 & -4.69 & -0.0644 & 0.0567 & -0.0054 & 0.0611 \\
\hline $5 C$ & -5.06 & -3.96 & -0.0548 & 0.0339 & -0.0043 & 0.0489 \\
\hline $6 C$ & -0.26 & 0.01 & -0.0244 & -0.0181 & 0.0096 & 0.0130 \\
\hline $7 C$ & -0.04 & -0.23 & -0.0173 & -0.0168 & 0.0079 & 0.0107 \\
\hline $8 C$ & -0.02 & 0.08 & -0.0039 & 0.0003 & 0.0013 & 0.0003 \\
\hline $9 \mathrm{C}$ & -0.02 & 0.13 & -0.0029 & 0.0006 & 0.0017 & -0.0001 \\
\hline $10 \mathrm{C}$ & -0.02 & -0.05 & 0.0002 & 0.0001 & 0.0002 & 0.0002 \\
\hline $11 \mathrm{C}$ & -0.02 & -0.06 & -0.0003 & 0.0004 & 0.0000 & 0.0004 \\
\hline $12 \mathrm{C}$ & -0.10 & -0.04 & 0.0003 & 0.0016 & 0.0003 & 0.0014 \\
\hline $13 \mathrm{C}$ & 0.00 & -0.01 & -0.0001 & 0.0000 & 0.0001 & 0.0001 \\
\hline $14 \mathrm{C}$ & -12.52 & -9.92 & -0.1063 & 0.2541 & 0.0003 & 0.1435 \\
\hline $15 C$ & -12.95 & -9.52 & -0.1114 & 0.2506 & -0.0026 & 0.1419 \\
\hline $16 \mathrm{C}$ & -0.62 & -0.35 & 0.1831 & 0.1843 & 0.1033 & 0.1175 \\
\hline $17 \mathrm{C}$ & -2.11 & -1.73 & 0.1521 & 0.1854 & 0.0895 & 0.1199 \\
\hline $18 \mathrm{C}$ & -2.66 & -3.17 & -0.0041 & 0.1051 & 0.0277 & 0.0730 \\
\hline $19 \mathrm{C}$ & -3.40 & -3.58 & -0.0113 & 0.1010 & 0.0224 & 0.0744 \\
\hline $20 \mathrm{C}$ & 3.27 & 3.56 & 0.1269 & 0.0461 & 0.0792 & 0.0502 \\
\hline $21 C$ & 1.70 & 2.17 & 0.1183 & 0.0710 & 0.0727 & 0.0615 \\
\hline $22 \mathrm{C}$ & -0.27 & -0.03 & 0.0048 & 0.0067 & 0.0043 & 0.0074 \\
\hline $23 C$ & -0.23 & -0.01 & 0.0042 & 0.0073 & 0.0074 & 0.0108 \\
\hline $24 \mathrm{C}$ & -0.17 & 0.03 & 0.0000 & 0.0025 & 0.0004 & 0.0025 \\
\hline $25 \mathrm{C}$ & -0.02 & -0.02 & 0.0012 & 0.0015 & 0.0009 & 0.0014 \\
\hline $26 C$ & 0.16 & 0.06 & 0.0000 & -0.0009 & 0.0012 & 0.0000 \\
\hline $27 \mathrm{C}$ & 0.26 & -0.02 & 0.0000 & -0.0004 & 0.0015 & -0.0004 \\
\hline $28 \mathrm{C}$ & -0.01 & -0.01 & -0.0011 & -0.0012 & -0.0001 & -0.0002 \\
\hline $29 C$ & -0.02 & -0.02 & -0.0006 & -0.0004 & 0.0000 & 0.0000 \\
\hline $30 \mathrm{~N}$ & 1.35 & 1.87 & 0.0288 & -0.0186 & 0.0239 & 0.0086 \\
\hline $31 \mathrm{~N}$ & 1.91 & 1.93 & 0.0313 & -0.0272 & 0.0250 & 0.0041 \\
\hline $32 \mathrm{~N}$ & -0.07 & 0.14 & -0.0118 & -0.0209 & 0.0061 & 0.0069 \\
\hline $33 \mathrm{~N}$ & -0.14 & 0.22 & -0.0071 & -0.0184 & 0.0057 & 0.0079 \\
\hline $34 \mathrm{O}$ & -0.07 & -0.11 & 0.0000 & 0.0009 & 0.0000 & 0.0009 \\
\hline 350 & 0.00 & -0.01 & 0.0001 & 0.0002 & 0.0002 & 0.0002 \\
\hline $36 \mathrm{O}$ & -0.11 & -0.18 & 0.0004 & 0.0017 & 0.0005 & 0.0017 \\
\hline $37 \mathrm{O}$ & -0.01 & -0.03 & 0.0008 & 0.0012 & 0.0007 & 0.0009 \\
\hline $38 \mathrm{O}$ & -0.71 & -0.66 & 0.0017 & 0.0116 & 0.0026 & 0.0119 \\
\hline 390 & -0.02 & -0.03 & 0.0001 & 0.0003 & 0.0003 & 0.0005 \\
\hline $40 \mathrm{O}$ & -0.07 & 0.02 & 0.0020 & 0.0013 & 0.0018 & 0.0014 \\
\hline 410 & 0.00 & 0.04 & 0.0001 & 0.0001 & 0.0001 & 0.0001 \\
\hline $42 \mathrm{O}$ & 0.01 & 0.13 & 0.0005 & -0.0002 & 0.0005 & -0.0002 \\
\hline 430 & -0.02 & 0.04 & 0.0019 & 0.0009 & 0.0013 & 0.0005 \\
\hline $44 \mathrm{O}$ & -0.02 & -0.03 & 0.0008 & 0.0011 & 0.0006 & 0.0008 \\
\hline $45 \mathrm{O}$ & -0.01 & -0.03 & 0.0000 & 0.0000 & 0.0000 & 0.0000 \\
\hline $46 \mathrm{O}$ & -0.02 & -0.12 & 0.0002 & -0.0002 & 0.0001 & -0.0001 \\
\hline $47 \mathrm{O}$ & -0.02 & -0.06 & 0.0003 & 0.0007 & 0.0001 & 0.0005 \\
\hline
\end{tabular}




\section{Conclusions}

The eleven fixed RSH density functionals, which include CAM-B3LYP, LC-wPBE, M11, N12, M11L, MN12L, N12SX, MN12SX, wB97, wB97X and wB97XD, are examined to establish whether they fulfill the empirical KID procedure. The assessment is done by comparing the values from HOMO and LUMO calculations to those that the $\triangle \mathrm{SCF}$ technique for the Blue-G1 molecule generates. This is an intermediate melanoidin pigment that is of both academic and industrial interest. The study has observed that the range-separated and hybrid meta-NGA density functionals tend to be the most suited to meeting this goal. In this case, they emerge as suitable alternative to the density functionals once it is established that the behavior of the functionals are tuned using a gap-fitting procedure. They also exhibit a desirable prospect of benefiting future studies in understanding the chemical reactivity that colored melanoidins with larger molecular weights have when reducing sugars react with proteins and peptides.

From the results of this work, it becomes evident that it is easy to predict the sites of interaction of the Blue-G1 pigment under study. This would involve having DFT-based reactivity descriptors including Parr functions and dual descriptor calculations. Evidently, the descriptors were useful in characterizing and describing the preferred reactive sites. They were also useful in comprehensively explaining the reactivity of the molecules.

Furthermore, it is also possible to predict the maximum absorption wavelength for the Blue-G1 with considerable accuracy. The prediction would involve the MN12SX density functional beginning with the HOMO-LUMO gap instead of TDDFT calculations. Such a finding is particularly crucial considering the likelihood of it being used to inform the alternative determination method on the color of larger systems such as prosthetic chromophore groups. Such becomes necessary in circumstance where it would not be possible to afford the TDDFT calculations.

Thus, it can be concluded that the model chemistry MN12SX/Def2TZVP/SMD(water) is the best combination of density functional, basis set and solvation model for the prediction of maximum absorption wavelength (starting from the KS HOMO-LUMO gap) and for the prediction of the chemical reactivity sites for the Blue-G1 melanoidin pigment, and it is the recommended methodology to follow in future studies of analog compounds.

Supplementary Materials: Supplementary materials are available online.

Acknowledgments: This work has been partially supported by CIMAV, SC and Consejo Nacional de Ciencia y Tecnología (CONACYT, Mexico) through Grant 219566-2014 for Basic Science Research. Daniel Glossman-Mitnik conducted this work while a Visiting Lecturer at the University of the Balearic Islands from which support is gratefully acknowledged. This work was co-funded by the Ministerio de Economía y Competitividad (MINECO) and the European Fund for Regional Development (FEDER) (CTQ2014-55835-R).

Author Contributions: Daniel Glossman-Mitnik conceived of and designed the research and headed, wrote and revised the manuscript, while Juan Frau contributed to the writing and the revision of the article.

Conflicts of Interest: The authors declare no conflict of interest regarding the publication of this paper.

\section{References}

1. Argirova, M.D. Photosensitizer Activity of Model Melanoidins. J. Agric. Food Chem. 2005, 53, 1210-1214.

2. Glossman-Mitnik, D. A Comparison of the Chemical Reactivity of Naringenin Calculated with M06 Family of Density Functionals. Chem. Cent. J. 2013, 7, 155-161.

3. Salgado-Morán, G.; Ruiz-Nieto, S.; Gerli-Candia, L.; Flores-Holguín, N.; Favila-Pérez, A.; Glossman-Mitnik, D. Computational Nanochemistry Study of the Molecular Structure and Properties of Ethambutol. J. Mol. Model. 2013, 19, 3507-3515.

4. Martínez-Araya, J.I.; Salgado-Morán, G.; Glossman-Mitnik, D. Computational Nanochemistry Report on the Oxicams-Conceptual DFT and Chemical Reactivity. J. Phys. Chem. B 2013, 117, 6639-6651.

5. Glossman-Mitnik, D. Computational Nanochemistry Study of the Chemical Reactivity Properties of the Rhodamine B Molecule. Procedia Comput. Sci. 2013, 18, 816-825. 
6. Martínez-Araya, J.I.; Salgado-Morán, G.; Glossman-Mitnik, D. Computational Nutraceutics: Chemical Reactivity Properties of the Flavonoid Naringin by Means of Conceptual DFT. J. Chem. 2013, 2013, 850297.

7. Cervantes-Navarro, F.; Glossman-Mitnik, D. Density Functional Study of the Effects of Substituents on the Chemical Reactivity of the Indigo Molecule. J. Theor. Comput. Chem. 2013, 12, 1350013.

8. Alvarado-González, M.; Flores-Holguín, N.; Glossman-Mitnik, D. Computational Nanochemistry Study of the Molecular Structure and Properties of the Chlorophyll a Molecule. Int. J. Photoenergy 2013, 2013, 424620.

9. Glossman-Mitnik, D. Chemical Reactivity Theory within DFT Applied to the Study of the Prunin Flavonoid. Eur. Int. J. Sci. Technol. 2014, 3, 195-207.

10. Glossman-Mitnik, D. Computational Chemistry of Natural Products: A Comparison of the Chemical Reactivity of Isonaringin Calculated with the M06 Family of Density Functionals. J. Mol. Model. 2014, 20, 2316.

11. Glossman-Mitnik, D. Computational Nanochemistry Study of the Molecular Structure, Spectra and Chemical Reactivity Properties of the BFPF Green Fluorescent Protein Chromophore. In Biosensors Nanotechnology; Tiwari, A., Turner, A.P., Eds.; John Wiley \& Sons: Hoboken, NY, USA, 2014; pp. 201-238.

12. Glossman-Mitnik, D. Computational Nanochemistry Report of the Molecular Structure, Spectra and Chemical Reactivity Properties of Pheophorbide A. In Design and Applications of Nanomaterials for Sensors; Seminario, J.M., Ed.; Springer Science + Business Media: Dordrecht, The Netherlands, 2014; pp. 359-402.

13. Martínez-Araya, J.I.; Grand, A.; Glossman-Mitnik, D. Towards the Rationalization of Catalytic Activity Values by Means of Local Hyper-Softness on the Catalytic Site: A Criticism About the Use of Net Electric Charges. Phys. Chem. Chem. Phys. 2015, 17, 29764-29775.

14. Soto-Rojo, R.; Baldenebro-López, J.; Glossman-Mitnik, D. Study of Chemical Reactivity in Relation to Experimental Parameters of Efficiency in Coumarin Derivatives for Dye Sensitized Solar Cells Using DFT. Phys. Chem. Chem. Phys. 2015, 17, 14122-14129.

15. Martínez-Araya, J.I.; Glossman-Mitnik, D. The Substituent Effect from the Perspective of Local HyperSoftness. An Example Applied on Normeloxicam, Meloxicam and 4-Meloxicam: Non-Steroidal AntiInflammatory Drugs. Chem. Phys. Lett. 2015, 618, 162-167.

16. Frau, J.; Muñoz, F.; Glossman-Mitnik, D. Validation of the Koopmans' Theorem by Means of the Calculation of the Conceptual DFT Descriptors of Three Fluorescent DNA Staining Dyes. Chem. Inform. 2016, 2, 1-7.

17. Frau, J.; Muñoz, F.; Glossman-Mitnik, D. A Molecular Electron Density Theory Study of the Chemical Reactivity of cis- and trans-Resveratrol. Molecules 2016, 21, 1650.

18. Frau, J.; Muñoz, F.; Glossman-Mitnik, D. A Theoretical Study of the Chemical Reactivity of Neohesperidin Dihydrochalcone Through Conceptual DFT Descriptors. SDRP J. Comput. Chem. Mol. Model. 2016, 1, doi:10.25177/JCCMM.1.2.3.

19. Mendoza-Huízar, L.H.; Salgado-Morán, G.; Ramírez-Tagle, R.; Glossman-Mitnik, D. A Theoretical Quantum Study of the Intramolecular Interactions and Chemical Reactivity of Polymorphs A and B of Famotidine in the Gas, DMSO, and Aqueous Phases. Comput. Theor. Chem. 2016, 1075, 54-62.

20. Frau, J.; Muñoz, F.; Glossman-Mitnik, D. A Comparison of the Minnesota Family of Density Functionals for the Calculation of Conceptual DFT Descriptors: Citrus Flavonoids as a Test Case. Res. J. Chem. Sci. 2017, 7, 46-58.

21. Frau, J.; Glossman-Mitnik, D. A Comparative Study of the Glycating Power of Simple Carbohydrates in the Maillard Reaction by Means of Conceptual DFT Descriptors. Bri. J. Appl. Sci. Technol. 2017, 21, 32795.

22. Frau, J.; Muñoz, F.; Glossman-Mitnik, D. A Conceptual DFT Study of the Chemical Reactivity of Magnesium Octaethylprphyrin (MgOEP) as Predicted by the Minnesota Family of Density Functionals. Quím. Nova 2017, 40, 402-406.

23. Frau, J.; Glossman-Mitnik, D. Pyridoxamine Derivatives as Non Enzymatic Glycation Inhibitors: The Conceptual DFT Viewpoint. Res. J. Life Sci. Bioinform. Pharm. Chem. Sci. 2017, 2, 103-122.

24. Frau, J.; Glossman-Mitnik, D. Molecular Modeling Study of the Structures, Properties and Glycating Power of Some Reducing Disacharides. MOJ Drug Des. Dev. Ther. 2017, 1, 00003.

25. Frau, J.; Glossman-Mitnik, D. Computational Prediction of the Reactivity sites of Alzheimer Amyloid $\beta$-Peptides A $\beta 40$ and A $\beta 42$. ChemXpress 2017, 10, 120.

26. Frau, J.; Glossman-Mitnik, D. Conceptual DFT Descriptors of Amino Acids with Potential Corrosion Inhibition Properties Calculated with the Latest Minnesota Density Functionals. Front. Chem. 2017, 5, doi:10.3389/fchem.2017.00016. 
27. Sastre, S.; Frau, J.; Glossman-Mitnik, D. Computational Prediction of the Protonation Sites of Ac-Lys-(Ala)nLys-NH2 Peptides through Conceptual DFT and MEDT Descriptors. Molecules 2017, 22, 458.

28. Frau, J.; Ramis, R.; Glossman-Mitnik, D. Computational Prediction of the Preferred Glycation Sites of Model Helical Peptides Derived from Human Serum Albumin (HSA) and Lysozyme Helix 4 (LH4). Theor. Chem. Acc. 2017, 136, 39.

29. Frau, J.; Muñoz, F.; Glossman-Mitnik, D. Application of DFT Concepts to the Study of the Chemical Reactivity of Some Resveratrol Derivatives Through the Assessment of the Validity of the "Koopmans in DFT" (KID) Procedure. J. Theor. Comput. Chem. 2017, 16, 1750006.

30. Frau, J.; Glossman-Mitnik, D. Chemical Reactivity Theory Study of Advanced Glycation Endproduct Inhibitors. Molecules 2017, 22, 226.

31. Frau, J.; Glossman-Mitnik, D. A Conceptual DFT Study of the Molecular Properties of Glycating Carbonyl Compounds. Chem. Cent. J. 2017, 11, 8.

32. Frau, J.; Hernández-Haro, N.; Glossman-Mitnik, D. Computational Prediction of the pKas of Small Peptides through Conceptual DFT Descriptors. Chem. Phys. Lett. 2017, 671, 138-141.

33. Nursten, H. (Ed.) The Maillard Reaction-Chemistry, Biochemistry and Implications; The Royal Society of Chemistry: Cambridge, UK, 2005.

34. Ono, Y.; Watanabe, H.; Hayase, F. Identification of the Blue Pigment Formed in a D-Glucose-Glycine Reaction System. Bioscience, Biotechnology and Biochemistry 2010, 74, 2526-2528.

35. Parr, R.; Yang, W. Density-Functional Theory of Atoms and Molecules; Oxford University Press: New York, NY, USA, 1989.

36. Geerlings, P.; De Proft, F.; Langenaeker, W. Conceptual Density Functional Theory. Chem. Rev. 2003, 103, 1793-1873.

37. Toro-Labbé, A. (Ed.) Theoretical Aspects of Chemical Reactivity; Elsevier Science: Amsterdam, The Netherlands, 2007.

38. Chattaraj, P. (Ed.) Chemical Reactivity Theory - A Density Functional View; CRC Press. Taylor \& Francis Group: Boca Raton, FL, USA, 2009.

39. Domingo, L.R. Molecular Electron Density Theory: A Modern View of Reactivity in Organic Chemistry. Molecules 2016, 21, 1319.

40. Domingo, L.R.; Ríos-Gutiérrez, M.; Pérez, P. Applications of the Conceptual Density Functional Theory Indices to Organic Chemistry Reactivity. Molecules 2016, 21, 748.

41. Jasinski, R. Searching for Zwitterionic Intermediates in Hetero Diels-Alder Reactions between Methyl ג,p-dinitrocinnamate and Vinyl-Alkyl Ethers. Comput. Theor. Chem. 2014, 1046, 93-98.

42. Jasinski, R.; Mroz, K.; Kacka, A. Experimental and Theoretical DFT Study on Synthesis of Sterically Crowded 2,3,3,(4)5-Tetrasubstituted-4-nitroisoxazolidines via 1,3-Dipolar Cycloaddition Reactions Between Ketonitrones and Conjugated Nitroalkenes. J. Heterocycl. Chem. 2016, 53, 1424-1429.

43. Jasinski, R.; Jasinska, E.; Dresler, E. A DFT Computational Study of the Molecular Mechanism of [3 + 2] Cycloaddition Reactions between Nitroethene and Benzonitrile N-oxides. J. Mol. Model. 2017, 23, 13.

44. Huzinaga, S.; Andzelm, J.; Klobukowski, M.; Radzio-Audzelm, E.; Sakai, Y.; Tatewaki, H. Gaussian Basis Sets for Molecular Calculations; Elsevier: Amsterdam, The Netherlands, 1984.

45. Easton, R.; Giesen, D.; Welch, A.; Cramer, C.; Truhlar, D. The MIDI! Basis Set for Quantum Mechanical Calculations of Molecular Geometries and Partial Charges. Theor. Chem. Acc. 1996, 93, 281-301.

46. Lewars, E. Computational Chemistry -Introduction to the Theory and Applications of Molecular and Quantum Mechanics; Kluwer Academic Publishers: Dordrecht, The Netherlands, 2003.

47. Young, D. Computational Chemistry-A Practical Guide for Applying Techniques to Real-World Problems; John Wiley \& Sons: New York, NY, USA, 2001.

48. Jensen, F. Introduction to Computational Chemistry, 2nd ed.; John Wiley \& Sons: Chichester, UK, 2007.

49. Cramer, C. Essentials of Computational Chemistry - Theories and Models, 2nd ed.; John Wiley \& Sons: Chichester, UK, 2004.

50. Gledhill, J.D.; De Proft, F.; Tozer, D.J. Range-Separation Parameter in Tuned Exchange-Correlation Functionals: Successive Ionizations and the Fukui Function. J. Chem. Theory Comput. 2016, 12, 4879-4884.

51. Jacquemin, D.; Moore, B.; Planchat, A.; Adamo, C.; Autschbach, J. Performance of an Optimally Tuned Range-Separated Hybrid Functional for 0-0 Electronic Excitation Energies. J. Chem. Theory Comput. 2014, $10,1677-1685$. 
52. Parr, R.; Yang, W. Density Functional Approach to the Frontier-Electron Theory of Chemical Reactivity. J. Am. Chem. Soc. 1984, 106, 4049-4050.

53. Parr, R.; Szentpaly, L.; Liu, S. Electrophilicity Index. J. Am. Chem. Soc. 1999, 121, 1922-1924.

54. Gázquez, J.; Cedillo, A.; Vela, A. Electrodonating and Electroaccepting Powers. J. Phys. Chem. A 2007, 111, 1966-1970.

55. Chattaraj, P.; Chakraborty, A.; Giri, S. Net Electrophilicity. J. Phys. Chem. A 2009, 113, 10068-10074.

56. Morell, C.; Grand, A.; Toro-Labbé, A. New Dual Descriptor for Chemical Reactivity. J. Phys. Chem. A 2005, 109, 205-212.

57. Morell, C.; Grand, A.; Toro-Labbé, A. Theoretical Support for Using the $\Delta f(\mathbf{r})$ Descriptor. Chem. Phys. Lett. 2006, 425, 342-346.

58. Cárdenas, C.; Rabi, N.; Ayers, P.; Morell, C.; Jaramillo, P.; Fuentealba, P. Chemical Reactivity Descriptors for Ambiphilic Reagents: Dual Descriptor, Local Hypersoftness, and Electrostatic Potential. J. Phys. Chem. A 2009, 113, 8660-8667.

59. Ayers, P.; Morell, C.; De Proft, F.; Geerlings, P. Understanding the Woodward-Hoffmann Rules by Using Changes in Electron Density. Chem. A Eur. J. 2007, 13, 8240-8247.

60. Morell, C.; Ayers, P.; Grand, A.; Gutiérrez-Oliva, S.; Toro-Labbé, A. Rationalization of the Diels-Alder Reactions through the Use of the Dual Reactivity Descriptor $\Delta f(\mathbf{r})$. Phys. Chem. Chem. Phys. 2008, 10, 7239-7246.

61. Morell, C.; Hocquet, A.; Grand, A.; Jamart-Grégoire, B. A Conceptual DFT Study of Hydrazino Peptides: Assessment of the Nucleophilicity of the Nitrogen Atoms by Means of the Dual Descriptor $\Delta f(\mathbf{r})$. J. Mol. Struct. THEOCHEM 2008, 849, 46-51.

62. Domingo, L.R.; Pérez, P.; Sáez, J. Understanding the Local Reactivity in Polar Organic Reactions through Electrophilic and Nucleophilic Parr Functions. RSC Adv. 2013, 3, 1486-1494.

63. Chamorro, E.; Pérez, P.; Domingo, L.R. On the Nature of Parr Functions to Predict the Most Reactive Sites along Organic Polar Reactions. Chem. Phys. Lett. 2013, 582, 141-143.

64. Frisch, M.J.; Trucks, G.W.; Schlegel, H.B.; Scuseria, G.E.; Robb, M.A.; Cheeseman, J.R.; Scalmani, G.; Barone, V.; Mennucci, B.; Petersson, G.A.; et al. Gaussian 09 Revision D.01; Gaussian Inc.: Wallingford, CT, USA, 2009.

65. Weigend, F.; Ahlrichs, R. Balanced Basis Sets of Split Valence, Triple Zeta Valence and Quadruple Zeta Valence Quality for H to Rn: Design and Assessment of Accuracy. Phys. Chem. Chem. Phys. 2005, 7, 3297-3305.

66. Weigend, F. Accurate Coulomb-fitting Basis Sets for H to R. Phys. Chem. Chem. Phys. 2006, 8, 1057-1065.

67. Yanai, T.; Tew, D.P.; Handy, N.C. A New Hybrid Exchange-Correlation Functional Using the CoulombAttenuating Method (CAM-B3LYP). Chem. Phys. Lett. 2004, 393, 51-57.

68. Henderson, T.M.; Izmaylov, A.F.; Scalmani, G.; Scuseria, G.E. Can Short-Range Hybrids Describe Long-RangeDependent Properties? J. Chem. Phys. 2009, 131, 044108.

69. Peverati, R.; Truhlar, D.G. Improving the Accuracy of Hybrid Meta-GGA Density Functionals by Range Separation. J. Phys. Chem. Lett. 2011, 2, 2810-2817.

70. Peverati, R.; Truhlar, D.G. M11-L: A Local Density Functional That Provides Improved Accuracy for Electronic Structure Calculations in Chemistry and Physics. J. Phys. Chem. Lett. 2012, 3, 117-124.

71. Peverati, R.; Truhlar, D.G. An Improved and Broadly Accurate Local Approximation to the ExchangeCorrelation Density Functional: the MN12-L Functional for Electronic Structure Calculations in Chemistry and Physics. Phys. Chem. Chem. Phys. 2012, 14, 13171-13174.

72. Peverati, R.; Truhlar, D.G. Screened-Exchange Density Functionals with Broad Accuracy for Chemistry and Solid-State Physics. Phys. Chem. Chem. Phys. 2012, 14, 16187-16191.

73. Peverati, R.; Truhlar, D.G. Exchange-Correlation Functional with Good Accuracy for Both Structural and Energetic Properties while Depending Only on the Density and Its Gradient. J. Chem. Theory Comput. 2012, 8, 2310-2319.

74. Chai, J.; Head-Gordon, M. Systematic Optimization of Long-Range Corrected Hybrid Density Functionals. J. Chem. Phys. 2008, 128, 084106.

75. Chai, J.; Head-Gordon, M. Long-Range Corrected Hybrid Density Functionals with Damped Atom-Atom Dispersion Corrections. Phys. Chem. Chem. Phys. 2008, 10, 6615-6620.

76. Marenich, A.; Cramer, C.; Truhlar, D. Universal Solvation Model Based on Solute Electron Density and a Continuum Model of the Solvent Defined by the Bulk Dielectric Constant and Atomic Surface Tensions. J. Phys. Chem. B 2009, 113, 6378-6396. 
77. Halgren, T.A. Merck Molecular Force Field. I. Basis, Form, Scope, Parameterization, and Performance of MMFF94. J. Comput. Chem. 1996, 17, 490-519.

78. Halgren, T.A. Merck Molecular Force Field. II. MMFF94 van der Waals and Electrostatic Parameters for Intermolecular Interactions. J. Comput. Chem. 1996, 17, 520-552.

79. Halgren, T.A. MMFF VI. MMFF94s Option for Energy Minimization Studies. J. Comput. Chem. 1999, 20, 720-729.

80. Halgren, T.A.; Nachbar, R.B. Merck Molecular Force Field. IV. Conformational Energies and Geometries for MMFF94. J. Comput. Chem. 1996, 17, 587-615.

81. Halgren, T.A. Merck Molecular Force field. V. Extension of MMFF94 Using Experimental Data, Additional Computational Data, and Empirical Rules. J. Comput. Chem. 1996, 17, 616-641.

82. Egger, D.A.; Weissman, S.; Refaely-Abramson, S.; Sharifzadeh, S.; Dauth, M.; Baer, R.; Kümmel, S.; Neaton, J.B.; Zojer, E.; Kronik, L. Outer-Valence Electron Spectra of Prototypical Aromatic Heterocycles From an Optimally Tuned Range-Separated Hybrid Functional. J. Chem. Theory Comput. 2014, 10, 1934-1952.

83. Foster, M.E.; Wong, B.M. Nonempirically Tuned Range-Separated DFT Accurately Predicts Both Fundamental and Excitation Gaps in DNA and RNA Nucleobases. J. Chem. Theory Comput. 2012, 8, 2682-2687.

84. Foster, M.E.; Azoulay, J.D.; Wong, B.M.; Allendorf, M.D. Novel Metal-Organic Framework Linkers for Light Harvesting Applications. Chem. Sci. 2014, 5, 2081-2090.

85. Karolewski, A.; Stein, T.; Baer, R.; Kümmel, S. Communication: Tailoring the Optical Gap in Light-Harvesting Molecules. J. Chem. Phys. 2011, 134, 151101.

86. Karolewski, A.; Kronik, L.; Kümmel, S. Using Optimally Tuned Range Separated Hybrid Functionals in Ground-State Calculations: Consequences and Caveats. J. Chem. Phys. 2013, 138, 204115.

87. Koppen, J.V.; Hapka, M.; Szczeniak, M.M.; Chalasinski, G. Optical Absorption Spectra of Gold Clusters $\mathrm{Au}(\mathrm{n})(\mathrm{n}=4,6,8,12,20)$ From Long-Range Corrected Functionals with Optimal Tuning. J. Chem. Phys. 2012, 137, 114302.

88. Kronik, L.; Stein, T.; Refaely-Abramson, S.; Baer, R. Excitation Gaps of Finite-Sized Systems from Optimally Tuned Range-Separated Hybrid Functionals. J. Chem. Theory Comput. 2012, 8, 1515-1531.

89. Kuritz, N.; Stein, T.; Baer, R.; Kronik, L. Charge-Transfer-Like $\pi \rightarrow \pi^{*}$ Excitations in Time-Dependent Density Functional Theory: A Conundrum and Its Solution. J. Chem. Theory Comput. 2011, 7, 2408-2415.

90. Lima, I.T.; Prado, A.D.S.; Martins, J.B.L.; de Oliveira Neto, P.H.; Ceschin, A.M.; da Cunha, W.F.; da Silva Filho, D.A. Improving the Description of the Optical Properties of Carotenoids by Tuning the LongRange Corrected Functionals. J. Phys. Chem. A 2016, 120, 4944-4950.

91. Manna, A.K.; Lee, M.H.; McMahon, K.L.; Dunietz, B.D. Calculating High Energy Charge Transfer States Using Optimally Tuned Range-Separated Hybrid Functionals. J. Chem. Theory Comput. 2015, 11, 1110-1117.

92. Moore, B., II; Autschbach, J. Longest-Wavelength Electronic Excitations of Linear Cyanines: The Role of Electron Delocalization and of Approximations in Time-Dependent Density Functional Theory. J. Chem. Theory Comput. 2013, 9, 4991-5003.

93. Niskanen, M.; Hukka, T.I. Modeling of Photoactive Conjugated Donor-Acceptor Copolymers: The Effect of the Exact HF Exchange in DFT Functionals on Geometries and Gap Energies of Oligomer and Periodic Models. Phys. Chem. Chem. Phys. 2014, 16, 13294-13305.

94. Pereira, T.L.; Leal, L.A.; da Cunha, W.F.; Timóteo de Sousa Júnior, R.; Ribeiro Junior, L.A.; Antonio da Silva Filho, D. Optimally Tuned Functionals Improving the Description of Optical and Electronic Properties of the Phthalocyanine Molecule. J. Mol. Model. 2017, 23, 71.

95. Phillips, H.; Zheng, S.; Hyla, A.; Laine, R.; Goodson, T., III; Geva, E.; Dunietz, B.D. Ab Initio Calculation of the Electronic Absorption of Functionalized Octahedral Silsesquioxanes via Time-Dependent Density Functional Theory with Range-Separated Hybrid Functionals. J. Phys. Chem. A 2012, 116, 1137-1145.

96. Phillips, H.; Geva, E.; Dunietz, B.D. Calculating Off-Site Excitations in Symmetric Donor-Acceptor Systems via Time-Dependent Density Functional Theory with Range-Separated Density Functionals. J. Chem. Theory Comput. 2012, 8, 2661-2668.

97. Refaely-Abramson, S.; Baer, R.; Kronik, L. Fundamental and Excitation Gaps in Molecules of Relevance for Organic Photovoltaics from an Optimally Tuned Range-Separated Hybrid Functional. Phys. Rev. B 2011, 84, 075144-8.

98. Stein, T.; Kronik, L.; Baer, R. Prediction of Charge-Transfer Excitations in Coumarin-Based Dyes Using a Range-Separated Functional Tuned from First Principles. J. Chem. Phys. 2009, 131, 244119. 
99. Stein, T.; Kronik, L.; Baer, R. Reliable Prediction of Charge Transfer Excitations in Molecular Complexes Using Time-Dependent Density Functional Theory. J. Am. Chem. Soc. 2009, 131, 2818-2820.

100. Sun, H.; Autschbach, J. Electronic Energy Gaps for $\pi$-Conjugated Oligomers and Polymers Calculated with Density Functional Theory. J. Chem. Theory Comput. 2014, 10, 1035-1047.

101. Becke, A.D. Vertical Excitation Energies From the Adiabatic Connection. J. Chem. Phys. 2016, 145, 194107.

102. Baerends, E.J.; Gritsenko, O.V.; van Meer, R. The Kohn-Sham Gap, the Fundamental Gap and the Optical Gap: The Physical Meaning of Occupied and Virtual Kohn-Sham Orbital Energies. Phys. Chem. Chem. Phys. 2013, 15, 16408-16425.

103. Van Meer, R.; Gritsenko, O.V.; Baerends, E.J. Physical Meaning of Virtual Kohn-Sham Orbitals and Orbital Energies: An Ideal Basis for the Description of Molecular Excitations. J. Chem. Theory Comput. 2014, $10,4432-4441$.

104. Zhurko, G.; Zhurko, D. Chemcraft program Revision 1.6; Zhurko, G.A., Ed.; Akzo Nobel Coatings Inc.: High Point, NC, USA, 2012.

105. Poater, A.; Duran, M.; Jaque, P.; Toro-Labbé, A.; Solà, M. Molecular Structure and Bonding of Copper Cluster Monocarbonyls CunCO (N=1-9). J. Phys. Chem. B 2006, 110, 6526-6536.

106. Martínez-Araya, J.I. Why is the Dual Descriptor a More Accurate Local Reactivity Descriptor than Fukui Functions? J. Math. Chem. 2015, 53, 451-465.

Sample Availability: Samples of the compounds are not available from the authors.

(C) 2018 by the authors. Licensee MDPI, Basel, Switzerland. This article is an open access article distributed under the terms and conditions of the Creative Commons Attribution (CC BY) license (http:/ / creativecommons.org/licenses/by/4.0/). 